\title{
First ionization potential of the heaviest actinide lawrencium, element 103
}

\author{
Tetsuya K. Sato ${ }^{1, a}$, Masato Asai ${ }^{1}$, Anastasia Borschevsky $^{2}$, Thierry Stora ${ }^{3}$, Nozomi Sato ${ }^{1}$, \\ Yusuke Kaneya ${ }^{1,4}$, Kazuaki Tsukada ${ }^{1}$, Christoph E. Düllmann ${ }^{5,6,7}$, Klaus Eberhardt ${ }^{5,7}$, \\ Ephraim Eliav ${ }^{8}$, Shinichi Ichikawa ${ }^{9}$, Uzi Kaldor ${ }^{8}$, Jens V. Kratz ${ }^{7}$, Sunao Miyashita ${ }^{10}$, \\ Yuichiro Nagame ${ }^{1}$, Kazuhiro Ooe ${ }^{11}$, Akihiko Osa ${ }^{1}$, Dennis Renisch ${ }^{7}$, Jörg Runke ${ }^{6}$, \\ Matthias Schädel ${ }^{1}$, Petra Thörle-Pospiech ${ }^{5,7}$, Atsushi Toyoshima ${ }^{1}$, and Norbert Trautmann ${ }^{7}$ \\ ${ }^{1}$ Japan Atomic Energy Agency, Tokai, Ibaraki 319-1195, Japan \\ ${ }^{2}$ The Van Swinderen Institute for Particle Physics and Gravity, University of Groningen, $9700 \mathrm{AB}$ \\ Groningen, The Netherlands \\ ${ }^{3}$ ISOLDE, CERN, CH-1211 Geneva 23, Switzerland \\ ${ }^{4}$ Graduate School of Science and Engineering, Ibaraki University, Mito, Ibaraki 310-8512, Japan \\ ${ }^{5}$ Helmholtz-Institut Mainz, 55099 Mainz, Germany \\ ${ }^{6}$ GSI Helmholtzzentrum für Schwerionenforschung, 64291 Darmstadt, Germany \\ ${ }^{7}$ Institut für Kernchemie, Johannes Gutenberg-Universität Mainz, 55099 Mainz, Germany \\ ${ }^{8}$ School of Chemistry, Tel Aviv University, 69978 Tel Aviv, Israel \\ ${ }^{9}$ Nishina Center for Accelerator-Based Science, RIKEN, 2-1 Hirosawa, Wako, Saitama 351-0198, \\ Japan \\ ${ }^{10}$ Graduate School of Science, Hiroshima University, Kagamiyama, Higashi-Hiroshima 739-8526, \\ Japan \\ ${ }^{11}$ Institute of Science and Technology, Niigata University, Niigata 910-2181, Japan
}

\begin{abstract}
The first ionization potential ( $\left.\mathrm{IP}_{1}\right)$ of element 103, lawrencium (Lr), has been successfully determined for the first time by using a newly developed method based on a surface ionization process. The measured $\mathrm{IP}_{1}$ value is $4.963_{0.07}^{0.08} \mathrm{eV}$. This value is the smallest among those of actinide elements and is in excellent agreement with the value of $4.963(15) \mathrm{eV}$ predicted by state-of-the-art relativistic calculations also performed in this work. Our results strongly support that the $\mathrm{Lr}$ atom has an electronic configuration of $[\mathrm{Rn}] 7 s^{2} 5 f^{14} 7 p_{1 / 2}^{1}$, which is influenced by strong relativistic effects. The present work provides a reliable benchmark for theoretical calculations and also opens the way for studies on atomic properties of heavy elements with atomic number $Z>100$. Moreover, the present achievement has triggered a controversy on the position of lutetium $(\mathrm{Lu})$ and $\mathrm{Lr}$ in the Periodic Table of Elements.
\end{abstract}

\section{Introduction}

The chemical properties of an element are primarily governed by the configuration of electrons in its valence shell. The relativistic effects on the electronic structure of heavy elements in the seventh row of the Periodic Table become so strong that in some cases even their ground-state configurations may differ from those expected from lighter elements in the same group [1].

\footnotetext{
${ }^{a}$ Corresponding author: sato.tetsuya@jaea.go.jp
}

(C) The Authors, published by EDP Sciences. This is an Open Access article distributed under the terms of the Creative Commons Attribution License 4.0 (http://creativecommons.org/licenses/by/4.0/). 
According to the actinide concept established by G.T. Seaborg [2], element 103, lawrencium (Lr), is placed at the tail end of the actinide series of the Periodic Table as the heaviest actinide element. By analogy with $\mathrm{Lu}$, which is the lanthanide homologue of $\mathrm{Lr}$ and has an electronic structure of $[\mathrm{Xe}] 4 f^{14} 6 s^{2} 5 d^{1}$, the electronic configuration of $\mathrm{Lr}$ would be expected to be $[\mathrm{Rn}] 5 f^{14} 7 s^{2} 6 d^{1}$. Theoretical relativistic calculations, however, have predicted that the ground-state configuration of $\mathrm{Lr}$ is $[\mathrm{Rn}] 5 f^{14} 7 s^{2} 7 p_{1 / 2}^{1}$ since the $7 p_{1 / 2}$ orbital would be stabilized below the $6 d$ orbital in Lr by strong relativistic effects [3]. Since this prediction came out about 40 years ago, $\mathrm{Lr}$ has attracted interest of theoretical and experimental chemists.

Different electronic configuration yields different chemical behaviour. If the ground state configuration of $\mathrm{Lr}$ has a $7 p_{1 / 2}$ valence electron, this would have implications of high volatility of Lr, as Eichler pointed out as a result of a semi-empirical consideration [4]. This prompted Jost and his colleagues to investigate the volatility of atomic Lr using a gas chromatographic technique [5]. In their experiment, no evidence for $\mathrm{Lr}$ as a volatile element with a $7 p_{1 / 2}$ configuration was found.

In practice, chemical investigation of heavy elements such as Lr poses some difficulties. Elements heavier than fermium (Fm, $Z=100)$ must be produced at accelerators using reactions of heavy ions with heavy target materials. Moreover, both half-lives and cross sections of the isotopes of the still heavier elements are rapidly decreasing. Thus, they are usually available in quantities of a few atoms only at a time. Consequently, beginning with about the end of the actinides, properties of the elements must be studied on an atom-at-atime scale. So far, it has been experimentally shown that $\mathrm{Lr}$ exhibits a stable +3 oxidation state in solution [6], and its ionic radius was evaluated as $88.6 \pm 0.3 \mathrm{pm}$ [7]. Based on the empirically developed "actinide concept", and in agreement with theoretical calculations, in today's Periodic Table, the actinide element series terminates with Lr. However, it has not yet been experimentally confirmed that $\mathrm{Lr}$ has appropriate properties as the last member of the actinide series.

\section{The first ionization potential of $\mathrm{Lr}$}

Experimental determination of the ground-state electronic structure of $\mathrm{Lr}$ could demonstrate the magnitude of the influence of relativistic effects and provide new insights into the chemical properties of the heaviest elements.

The first ionization potential $\left(\mathrm{IP}_{1}\right)$ is a fundamental physical and chemical property of an element, which is qualitatively defined as the amount of energy required to remove the valence electron of an atom to form a positive ion. Experimentally determined $\mathrm{IP}_{1}$, therefore, would give information on the outermost electronic orbital of the atom. In addition, $\mathrm{IP}_{1}$ is one of the few physical quantities which can be estimated directly by theoretical calculations. A precise and accurate experimental determination of $\mathrm{IP}_{1}$ can therefore benchmark electronic structure calculations.

$\mathrm{IP}_{1}$ values of weighable amounts of nuclear-reactor-produced heavy elements up to einsteinium (Es, $Z=99$ ) have been measured by resonance ionization mass spectrometry (RIMS) [8]. RIMS was also applied to an investigation of Fm with a sample of $2.7 \times 10^{10}$ atoms of ${ }^{255} \mathrm{Fm}$ (half-life $T_{1 / 2}=20.1 \mathrm{~h}$ ). In that experiment, the atomic level structure, but not the $\mathrm{IP}_{1}$, was determined [9]. Recently, resonance ionization laser ion source (RILIS) studies optimized for short-lived nuclides made it possible to determine $\mathrm{IP}_{1}$ of astatine (At, $Z=85)$ using ${ }^{199}$ At $\left(T_{1 / 2}=7.2 \mathrm{~s}\right)$ produced in the proton-induced spallation reaction of uranium [10]. $\mathrm{IP}_{1}$ values of heavy elements with $Z \geq 100$, however, could not be determined experimentally so far, as the produced amounts of the atoms were too low for the above techniques. 
A sufficiently long-lived and detectable isotope for chemical experiments with $\mathrm{Lr}$ is ${ }^{256} \mathrm{Lr}$ $\left(T_{1 / 2}=27 \mathrm{~s}\right)$. It is produced at a rate of one atom per several seconds in the fusion-evaporation reaction of a ${ }^{249} \mathrm{Cf}$ target with a ${ }^{11} \mathrm{~B}$ beam [11]. A new and highly efficient experimental method was thus required to determine an $\mathrm{IP}_{1}$ value of $\mathrm{Lr}$ with such short-lived $\mathrm{Lr}$ atoms and in such low amounts.

In order to determine the $\mathrm{IP}_{1}$ value of $\mathrm{Lr}$, we have developed a novel method based on the surface ionization technique. The surface ionization is an ionization process which takes place on a solid surface kept at high temperature $[12,13]$. Based on the Saha-Langmuir equation, an analytical model describes the surface ionization process in a hollow tube (cavity)-type ionsource. The ionization efficiency, $I_{\mathrm{eff}}$, of an element in a small hot cavity can be expressed as:

$$
I_{\text {eff }}=\frac{N \exp \left(\frac{\phi-\mathrm{IP}_{1}^{*}}{k T}\right)}{1+N \exp \left(\frac{\phi-I \mathrm{IP}_{1}^{*}}{k T}\right)},
$$

where $\phi$ is the work function which is material-dependent, $k$ the Bolzmann constant, $T$ the temperature of the ionizing surface, and $N$ a parameter that depends on the effective number of atom-surface interactions in the cavity. $\mathrm{IP}_{1}^{*}$, the effective $\mathrm{IP}_{1}$, is directly related to $\mathrm{IP}_{1}$ as:

$$
\mathrm{IP}^{*}=\mathrm{IP}_{1}-k T \ln \left(\frac{Q_{1}}{Q_{0}}\right),
$$

where $Q_{i}$ and $Q_{o}$ are the partition functions of electrons in an atom and an ion at a given temperature, respectively, which can be calculated using excitation energies and statistical weights of their ground and excited states. In the present study, tantalum (Ta) was chosen as a cavity material. The $\mathrm{IP}_{1}$ value of the element of interest is deduced from Eq. (1) where the parameter $N$ is determined from a set of experimental ionization efficiencies ( $\left.I_{\text {eff }}\right)$ of known $\mathrm{IP}_{1}$ of some elements [14].

A schematic experimental set-up used in the measurement is shown in Fig. 1. To investigate surface ionization behaviour of short-lived $\mathrm{Lr}$ and other isotopes, we employed an ISOL (Isotope Separator On-Line) system which has a surface ion-source coupled to an aerosol gas-jet transport system at the tandem accelerator facility of Japan Atomic Energy Agency (JAEA). The ion-source has been newly developed for this purpose. The set-up consists of a target recoil chamber, a gas-jet transport system [15], a surface ion source installed in the ISOL system [16], and $\alpha / \gamma$ spectrometry systems. For an efficient $\alpha$-particle measurement, we applied a rotating catcher wheel apparatus MANON (Measurement system of Alpha particle and spontaneous fission events ON-line) [17]. Nuclear reaction products produced in the target recoil chamber were attached on $\mathrm{CdI}_{2}$ aerosol flowing into the chamber with $1.4 \mathrm{~L} / \mathrm{min} \mathrm{He}$ carrier gas. The radioactivity-laden aerosol particles were transported continuously into the ion-source via a Teflon capillary. Surface-ionized nuclear reaction products were extracted from the ion source and accelerated for mass separation. After the mass separation, ions of the isotope of interest are collected and detected to determine the ionization efficiency. For ${ }^{256} \mathrm{Lr}, I_{\text {eff }}$ values of $(33 \pm 4) \%$ and $(36 \pm 7) \%$ were measured at $2700 \mathrm{~K}$ and $2800 \mathrm{~K}$, respectively [14].

To determine the value of the parameter $N$ in Eq. (1) at each temperature, short-lived lanthanide and alkali isotopes ${ }^{142,143} \mathrm{Eu},{ }^{143} \mathrm{Sm},{ }^{148} \mathrm{~Tb},{ }^{153,154} \mathrm{Ho},{ }^{157} \mathrm{Er},{ }^{162} \mathrm{Tm},{ }^{165} \mathrm{Yb},{ }^{168} \mathrm{Lu}$ and ${ }^{80} \mathrm{Rb}$ produced in nuclear reactions of ${ }^{11} \mathrm{~B}$ beams with mixed lanthanide targets and a Ge target were provided to the experimental set-up and their ionization efficiencies were measured. Figure 2 shows the $I_{\text {eff }}$ values at $2800 \mathrm{~K}$ as a function of $\mathrm{IP}_{1} *$. The $\mathrm{IP}_{1} *$ value for each element was calculated with Eq. (2) using energies and statistical weights of lowlying excited states in the ion and the atom of each element provided by the National Institute of Standards and Technology (NIST) atomic database [18]. The determined $N$ 


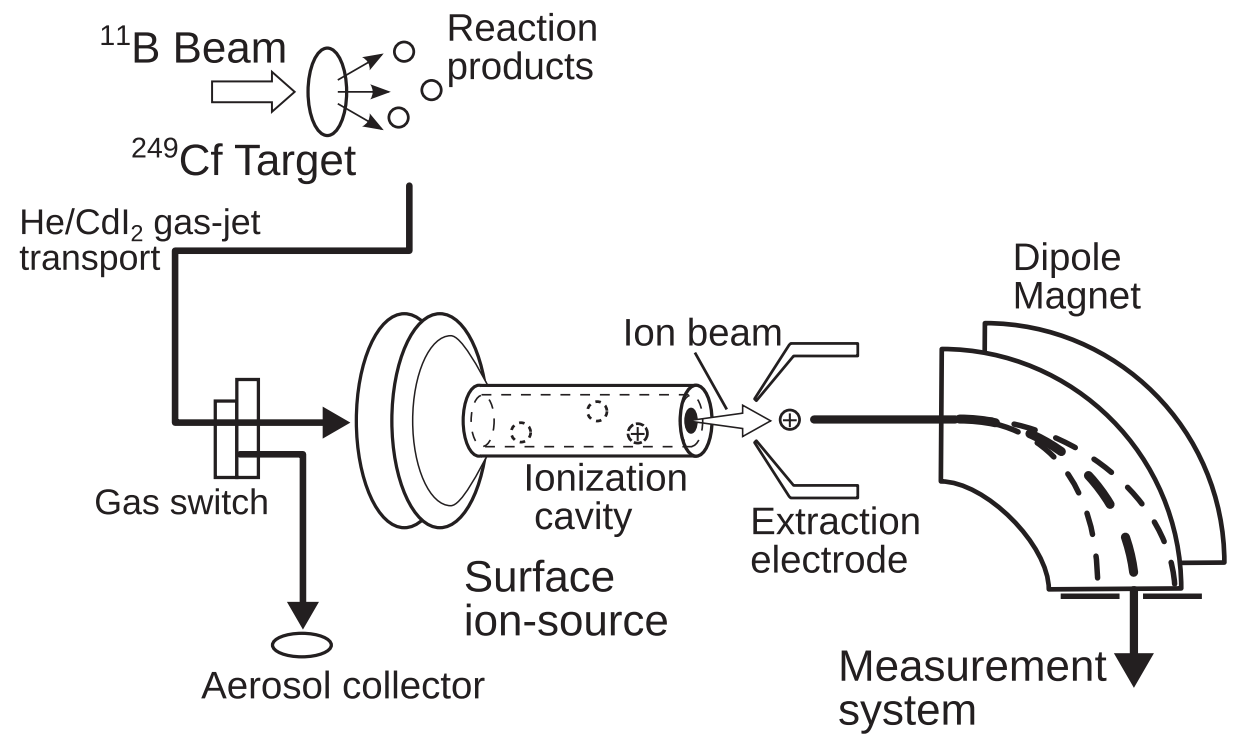

Figure 1. Schematic diagram of the experimental set-up for a measurement of the first ionization potential of Lr. The set-up was constructed based on the JAEA-ISOL system.

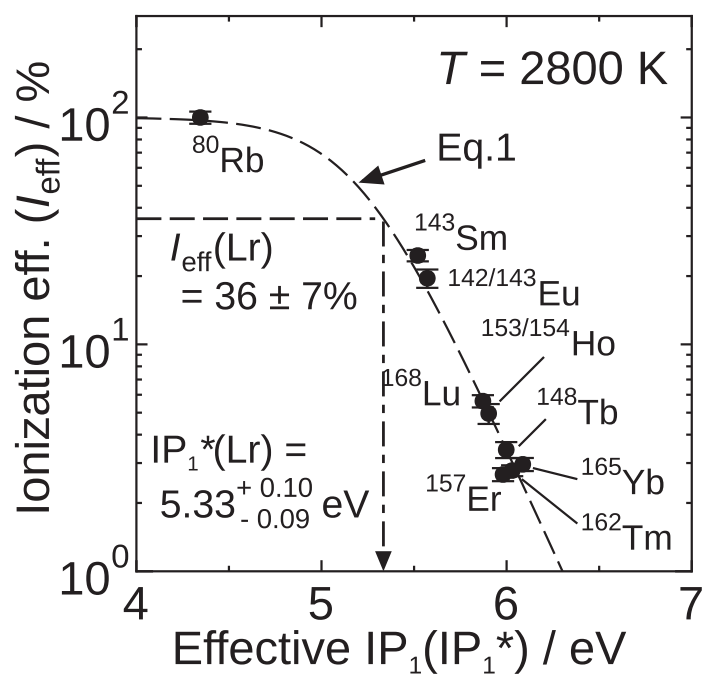

Figure 2. The ionization efficiency $\left(\mathrm{I}_{\mathrm{eff}}\right)$ of various short-lived isotopes as a function of the effective $\mathrm{IP}_{1}$ $\left(\mathrm{IP}_{1}^{*}\right)$ at $2800 \mathrm{~K}$. The dashed curve is obtained by fitting the experimental data using Eq. (1). The position of the measured Ieff value of $\operatorname{Lr},(36 \pm 4) \%$, is also shown. From the fitted Eq. (1) with $N=50 \pm 3$, $\mathrm{IP}_{1}^{*}$ of $\mathrm{Lr}$ is calculated to be $5.33_{-0.09}^{+0.10} \mathrm{eV}$. This corresponds to an $\mathrm{IP}_{1}$ value of $4.97_{-0.11}^{+0.13} \mathrm{eV}$ [14].

values at $T=2700 \mathrm{~K}$ and $2800 \mathrm{~K}$ were $43 \pm 3$ and $50 \pm 3$, respectively. Using the $N$ values, the $\mathrm{Lr} \mathrm{IP}_{1} *$ values at $T=2700 \mathrm{~K}$ and $2800 \mathrm{~K}$ were calculated to be $5.29_{-0.07}^{+0.08} \mathrm{eV}$ and $5.33_{-0.09}^{+0.10} \mathrm{eV}$ respectively. Since the excitation energies of $\mathrm{Lr}$ and $\mathrm{Lr}^{+}$, which are required to calculate $\mathrm{IP}_{1}$ using Eq. (2) are unavailable, we employed theoretical values proposed in [19]. Then, $\mathrm{IP}_{1}$ values of $4.95_{-0.08}^{+0.10}$ and $4.97_{-0.11}^{+0.13}$ were determined at $T=2700 \mathrm{~K}$ and $2800 \mathrm{~K}$, respectively, for Lr. Based on these results, our experimentally determined value for $\mathrm{IP}_{1}$ of $\mathrm{Lr}$ is $4.96_{-0.07}^{+0.08} \mathrm{eV}$. 


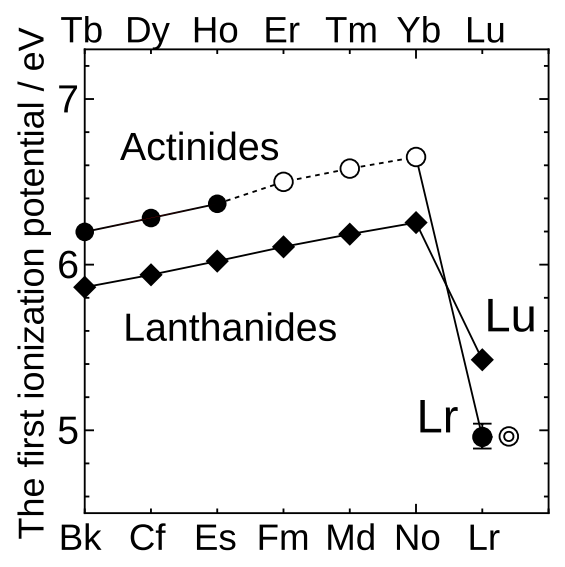

Figure 3. Variation of the experimental first ionization potentials of actinides $(\bullet)$ and lanthanides $(\bullet)$. Open circles indicate estimated values for the heavy actinides [20], while the double circle shows the theoretically calculated value [14].

A state-of-the-art relativistic calculation of the $\mathrm{IP}_{1}$ of $\mathrm{Lr}$ was also performed. The calculation was conducted using the relativistic coupled cluster approach with single, double, and perturbative triple excitations $(\mathrm{DC} \operatorname{CCSD}(\mathrm{T}))$, and corrected for the Breit contribution and Lamb shift. The calculated $\mathrm{IP}_{1}$ value is $4.963(15) \mathrm{eV}$. Our experimental result on the first ionization potential of $4.96_{-0.07}^{+0.08}$ is in excellent agreement with the theoretical value calculated in this work [14].

\section{Where is the appropriate place of $\mathrm{Lr}$ in the Periodic Table?}

The measured $\mathrm{IP}_{1}$ value of $\mathrm{Lr}, 4.96 \mathrm{eV}$, is shown together with the theoretical values and experimental and/or estimated values [20-22] of lanthanide and actinide elements in Fig. 3. It is known that the $\mathrm{IP}_{1}$ value increases monotonically from terbium to ytterbium and decreased dramatically at lutetium in the lanthanide series. As shown in Fig. 3, $\mathrm{IP}_{1}$ of $\mathrm{Lr}$ is distinctly lower than that of $\mathrm{Lu}, 5.425871(12) \mathrm{eV}$ [21] and the lowest among the actinides. This validates the position of $\mathrm{Lr}$ as the last actinide element ( $f$-block element) in the Periodic Table, and quantitatively reflects and confirms the theoretically predicted situation of closed $5 f^{14}$ and $7 s^{2}$ shells with an additional weakly-bound electron in the valence orbital. The $\mathrm{IP}_{1}$ of $\mathrm{Lr}$ is surprisingly low, with the valence electron even weaker bound than in sodium (5.1291 eV) [22]. The metallicity of Lr can be thus compared with an alkali metal.

On the other hand, our experimental result strongly supports that the outermost electronic orbital of $\mathrm{Lr}$ is the $7 p_{1 / 2}$, due to the influence of the strong relativistic effects. This proposed electronic configuration of $\mathrm{Lr},[\mathrm{Rn}] 5 f^{14} 7 s^{2} 7 p_{1 / 2}^{1}$, suggests it would also not be out of the place in the $p$-block. A transition element is defined as "an element whose atom has an incomplete $d$ sub-shell, or which can give rise to cations with an incomplete $d$ sub-shell" by the International Union of Pure and Applied Chemistry (IUPAC) [23]. According to this definition, Lr would not belong to the transition elements.

It was also suggested that $\mathrm{Lu}$ and $\mathrm{Lr}$ should be placed below scandium and yttrium in the $d$-block, based on its ground-state valence-electron configuration and self-consistent group trends for various chemical and physical properties [24].

Since the introduction of the "actinide concept" as the most dramatic modern revision of the Periodic Table in the 1940s, the element with atomic number 103, lawrencium, played a crucial role as the last element in the actinide series. This special position has placed this 
element into the focus of discussion on the influence of relativistic effects on electronic structure and motivated the determination of its atomic properties. Now, our study not only provides new information, both experimental and theoretical, but also poses a new question: "what are the appropriate positions of $\mathrm{Lu}$ and $\mathrm{Lr}$ in the Periodic Table?" This issue was already on the agenda of 48th IUPAC Council Meeting held in Korea in 2015 [25]. An IUPAC task group has been formed in order to make a recommendation regarding the membership of group 3 of the Periodic Table: Project No. 2015-039-2-200 [26].

We thank the JAEA tandem accelerator crew for supplying intense and stable beams for the experiments. The ${ }^{249} \mathrm{Cf}$ was made available by $\mathrm{H}$. Nitsche (University of California, Berkeley); it was produced in the form of ${ }^{249} \mathrm{Bk}$ through the former Transplutonium Element Production Program at Oak Ridge National Laboratory (ORNL) under the auspices of the Director, Office of Science, Office of Basic Energy Sciences, Chemical Sciences, Geosciences, and Biosciences Division of the US Department of Energy. Financial support by the Helmholtz-Institut Mainz is acknowledged. This work has been partly supported by the Grant-in-Aid for Scientific Research (C) no. 26390119 of the Ministry of Education, Science, Sports and Culture (MEXT).

\section{References}

[1] P. Pyykkö, Chem. Rev. 88, 563 (1988).

[2] G.T. Seaborg, Science 104, 379 (1946).

[3] J.-P. Desclaux and B. Fricke, J. Phys. 41, 943 (1980).

[4] B. Eichler and S. Hübener, Inorg. Chim. Acta, 146, 261 (1988).

[5] D.T. Jostet al., Inorg. Chim. Acta, 146, 255 (1988).

[6] R.J. Silva et al., lnorg. Nucl. Chem. Lett. 6, 733 (1970).

[7] D.C. Hoffmann et al., J. Radioanal. Nucl. Chem. 124, 135 (1988).

[8] J. Peterson et al., J. Alloy. Comp. 271-273, 876 (1998).

[9] M. Sewtz et al., Phys. Rev. Lett. 90, 163002 (2003).

[10] S. Rothe et al., Nature Comm. 4, 1835 (2013).

[11] N. Sato et al., Radiochim. Acta 102, 211 (2014).

[12] É.Y. Zandberg and N. Ionov, Sov. Phys. Usp. 2, 255 (1959).

[13] R. Kirchner, Nucl. Instr. Meth. A292, 203 (1990).

[14] T.K. Sato et al., Nature 520, 209 (2015).

[15] T.K. Sato et al., J. Radioanal. Nucl. Chem. 303, 1253 (2015).

[16] T.K. Sato et al., Rev. Sci. Instrum. 84, 023304(5) (2013).

[17] Y. Nagame et al., J. Nucl. Radiochem. Sci. 3, 85 (2002).

[18] A. Kramida et al., NIST Atomic Spectra Database version 5.1 (2013); http://physics.nist.gov/asd (accessed July 2014).

[19] A. Borschevsky et al., Eur. Phys. J. D45, 115 (2007).

[20] J. Sugar, J. Chem. Phys. 60, 4103 (1974).

[21] H. Maeda et al., J. Phys. B 22, L511 (1989).

[22] Handbook of Chemistry and Physics, 65th ed., eds. R.C. Weast, M.J. Astle, W.H. Beyer (CRC, 1985).

[23] IUPAC Gold book http: //goldbook.iupac.org/

[24] W.B. Jensen, Found Chem. 17, 23-31 (2015).

[25] 48th IUPAC Council Meeting Final Agenda Book.

[26] E. Scherri, Chem. International, March-April 22-23 (2016). 